\title{
Flavin Adenine Dinucleotide Status and the Effects of High-Dose Riboflavin Treatment in Short-Chain Acyl-CoA Dehydrogenase Deficiency
}

\author{
BIANCA T. vAN MALDEGEM, MARINUS DURAN, RONALD J. A. WANDERS, HANS R. WATERHAM, AND FRITS A. WIJBURG \\ Department of Pediatrics [B.T.M, F.A.W.] and Laboratory Genetic Metabolic Diseases [M.D., R.J.A.W., H.R.W.], University of \\ Amsterdam, 1105 AZ Amsterdam, the Netherlands
}

\begin{abstract}
Short-chain acyl-CoA dehydrogenase deficiency (SCADD) is an inborn error, biochemically characterized by increased plasma butyrylcarnitine $(\mathrm{C} 4-\mathrm{C})$ concentration and increased ethylmalonic acid (EMA) excretion and caused by rare mutations and/or common gene variants in the SCAD encoding gene. Although its clinical relevance is not clear, SCADD is included in most US newborn screening programs. Riboflavin, the precursor of flavin adenine dinucleotide (FAD, cofactor), might be effective for treating SCADD. We assessed the FAD status and evaluated the effects of riboflavin treatment in a prospective open-label cohort study involving 16 patients with SCADD, subdivided into mutation/mutation (mut/mut), mutation/variant (mut/var), and variant/variant (var/var) genotype groups. Blood FAD levels were normal in all patients before therapy, but significantly lower in the mut/var and var/var groups compared with the mut/mut group. Riboflavin treatment resulted in a decrease in EMA excretion in the mut/var group and in a subjective clinical improvement in four patients from this group. However, this improvement persisted after stopping treatment. These results indicate that high-dose riboflavin treatment may improve the biochemical features of SCADD, at least in patients with a mut/var genotype and low FAD levels. As our study could not demonstrate a clinically relevant effect of riboflavin, general use of riboflavin cannot be recommended. (Pediatr Res 67: 304-308, 2010)
\end{abstract}

$\mathrm{S}^{\mathrm{s}}$ hort-chain acyl-CoA dehydrogenase (SCAD, EC 1.3.99.2; MIM 606885) deficiency (SCADD; MIM 201470) is an autosomal recessive inborn error of mitochondrial fatty acid oxidation. SCADD is most frequently diagnosed as a result of investigations for developmental delay, epilepsy, behavioral disorders, hypoglycaemia, and hypotonia (1-13), but the diagnosis of SCADD probably has no clinical significance in many individuals (5). SCADD is one of the most common inborn errors of metabolism (5). Although SCADD does not seem to meet newborn screening criteria (5), a committee of the National Academy of Clinical Biochemistry could not reach consensus on recommending against adoption of SCADD in newborn screening programs (14). Therefore, SCADD is still included in newborn screening programs in

Received November 4, 2009; accepted November 10, 2009

Correspondence: Bianca T. van Maldegem, M.D., Department of Pediatrics, Academic Medical Center, University of Amsterdam, Meibergdreef 9, 1105 AZ Amsterdam, the Netherlands; e-mail: B.van.Maldegem@gelre.nl

Supported by "Metakids" (www.metakids.nl), the Dutch association for research on metabolic disorders. most US states (15). In addition, potential treatment options for SCADD have never been systematically studied.

SCADD is caused by decreased activity of the first enzyme of the short-chain fatty acid $\beta$-oxidation spiral, which catalyzes the dehydrogenation of butyryl-CoA (C4-CoA). When SCAD activity is impaired, C4-CoA will accumulate and is subsequently converted into different metabolites including 1$)$ the corresponding carnitine-ester, i.e. butyrylcarnitine (C4-C); 2) the corresponding glycine ester (butyrylglycine); 3) butyrate; and 4) ethylmalonic acid (EMA). C4-C can be measured in blood, whereas EMA can be measured in urine. In general, these two metabolites are both elevated in SCADD, although to different extents.

The diagnosis is confirmed by DNA analysis of the gene that encodes SCAD (ACADS) (5). Currently, 38 different mutations have been reported in patients with SCADD $(2,3,5,6,10,11,16-18)$. In addition, two common $A C A D S$ variants, c.511C $>\mathrm{T}$ and c.625G $>\mathrm{A}$ have been found in the general population with prevalence of homozygosity of $\sim 0.3$ and $5.5 \%$, respectively $(19,20)$. Most patients with SCADD are homozygous or compound heterozygous for one or two of the common $A C A D S$ variants or for a combination of one of these variants with an $A C A D S$ mutation $(2,3,5,10,11)$. Both gene variants may play a modifying role in the pathogenesis of clinical SCADD, by conferring susceptibility to clinical disease $(10,21)$.

The SCAD enzyme is a flavoprotein consisting of four subunits, each of which contains one molecule of its cofactor flavin adenine dinucleotide (FAD). Riboflavin, vitamin B2 (7,8-dimethyl-10-ribityl-isoalloxazine), is the precursor of FAD and is predominantly ingested through the consumption of milk and dairy products (22). FAD binding is important not only for the catalytic activity of flavoproteins but also for their folding, assembly, and/or stability (23-25). Because all of the studied ACADS mutations and variants result in protein misfolding (3), riboflavin therapy might be particularly efficacious in patients with SCADD if it can stabilize the affected protein. In addition, riboflavin deficiency is a relatively common con-

Abbreviations: ACADS, SCAD encoding gene; C4-C, butyrylcarnitine; EMA, ethylmalonic acid; FAD, flavin adenine dinucleotide; mut/mut, mutation/mutation; mut/var, mutation/variant; SCADD, short-chain acyl-CoA dehydrogenase deficiency; var/var, variant/variant 
dition (26) and could therefore be a common environmental factor reducing $S C A D$ activity in susceptible SCADD individuals, resulting in clinical disease. Profound riboflavin deficiency, typically presenting as angular stomatitis, cheilosis, and glossitis, is common in developing country populations $(27,28)$ but rare in Western societies $(29)$.

Results of riboflavin treatment have previously been reported in only three patients with SCADD $(12,13,30)$. In two of them, riboflavin treatment seemed to be beneficial $(12,30)$. In one of them, clinical improvement persisted after cessation of therapy (12).

The purpose of our study was to systematically assess the FAD status in individuals with SCADD and to evaluate the effects of high-dose riboflavin treatment on the biochemical characteristics and clinical status in a relatively large cohort of patients with SCADD and to compare effects between the different ACADS genotypes.

\section{SUBJECTS AND METHODS}

Study design and patients. We conducted a prospective open-label cohort study between January 2003 and January 2008. Sixteen patients with SCADD, all initially investigated because of clinical symptoms and diagnosed with SCADD after sequence analysis of all exons and flanking intronic sequences of the ACADS gene had been performed (Tables 1 and 2), were included in the study. All patients but patients 11 and 16 were part of the Dutch SCADD cohort that has been previously described (5). Patients were classified into three different genotype groups: 1) ACADS mutations on both alleles [mutation/mutation (mut/mut) group; $n=3$ ]; 2) an ACADS mutation on one allele and an $A C A D S$ variant on the other allele or both alleles [mutation/variant (mut/var) group; $n=8$ ]; and 3) $A C A D S$ variants on both alleles [variant/variant (var/var) group; $n=5$ ] (Table 1).

This study was approved by the Institutional Review Board of the Academic Medical Center and informed consent was obtained from all parents.

Treatment and assessment. Riboflavin was administered as $10 \mathrm{mg} / \mathrm{kg}$ body weight per day, divided into three doses with a maximum of $150 \mathrm{mg} / \mathrm{d}$, and ingested during meals. Before (d 0) and 5 wk after starting treatment (d 35), blood was obtained for $\mathrm{C} 4-\mathrm{C}$ and $\mathrm{FAD}$ analyses, and urine was obtained for determination of FAD excretion. During the $7 \mathrm{~d}$ preceding $\mathrm{d} 0$ and $\mathrm{d}$ 35 , five early morning voids were collected to determine EMA levels in urine. All urine and blood samples were obtained after an overnight fast and, for samples collected during riboflavin therapy, before the morning riboflavin dose.

Blood and urine analyses. The FAD status was determined by analysis of whole blood and urine using HPLC (31). Acylcarnitine profiles were determined using electrospray tandem mass spectrometry (32). Urine samples were stored at $-20^{\circ} \mathrm{C}$ until analysis and were analyzed for organic acids by gas chromatography/mass spectrometry (33).

Clinical signs and symptoms. Both before and $5 \mathrm{wk}$ after the start of riboflavin therapy, a medical history was taken. At the 5 wk appointment, parents were asked if they had noticed any changes in the condition of their child during the period of riboflavin treatment.

Statistical Analysis. The Kruskall-Wallis test was used to compare the three different genotype groups and the Mann-Whitney test to compare two different groups. The Wilcoxon signed rank test was used to compare baseline values with values obtained during treatment. The level of significance was set at $p<0.05$. Analyses were performed using Graphpad Prism 3.0.

\section{RESULTS}

FAD status and FAD response to riboflavin treatment. Blood FAD concentrations before riboflavin treatment were within the normal reference range in all but one patient (patient 2, who received high caloric tube feeding, Table 1). A significant difference in blood FAD concentrations was observed among the three genotype groups before the start of treatment, with the lowest FAD concentrations observed in the mut/var and var/var groups (Table 1). During treatment, me- dian blood and urine FAD concentrations in all the patients with SCADD increased significantly and did not differ between the three genotype groups (Table 1).

EMA and C4-C in response to riboflavin treatment. Median EMA excretion decreased significantly only in the mut/ var group (Table 1). None of the genotype groups showed a significant decrease in blood C4-C concentration (Table 1). However, in five of the eight patients in the mut/var group a biochemical response, defined as a clear decrease in plasma C4-C levels, in response to riboflavin was observed (Table 1). In four of them, this $\mathrm{C} 4-\mathrm{C}$ decrease was accompanied by a distinct decrease in EMA excretion. Of note, EMA levels in the fifth patient were only slightly increased before treatment (Table 1). In three of these five biochemically responding patients, blood FAD levels were measured before and during riboflavin treatment. The increase in FAD levels was significantly higher compared with the increase in plasma FAD levels in the patients with SCADD who did not respond to riboflavin (Table 1).

Clinical signs and symptoms. During treatment, all patients (and/or their parents) reported a change in color of the urine. In four of the patients (patients 4, 7, 8, and 10), all responding biochemically to riboflavin, a slight clinical improvement was reported $5 \mathrm{wk}$ after initiation of riboflavin treatment (Table 2). The improved clinical condition persisted in all four patients after discontinuation of treatment and during a follow-up of $2 \mathrm{y}$.

\section{DISCUSSION}

Our study is the first to systematically examine the efficacy of a potential treatment for SCADD, one of the more common inborn errors of metabolism. SCADD is included in newborn screening programs in most of the US states, although it has questionable clinical relevance and no treatment has been evaluated.

A high dose of riboflavin $\left(10 \mathrm{mg} \cdot \mathrm{kg}^{-} \cdot \mathrm{d}^{-}\right.$, with a maximum of $150 \mathrm{mg} / \mathrm{d}$ ) was administered to patients in this study. This dose was thought to be sufficient to obtain the maximal attainable FAD levels in these patients, as there is little additional absorption of riboflavin for single doses greater than $30 \mathrm{mg}$ (34). The significant increase during treatment in blood FAD concentrations and urinary FAD excretion in the total study group supports this (Table 1).

None of the patients had decreased blood FAD concentrations at baseline. However, the median blood FAD concentrations in the mut/var and var/var groups were significantly lower compared with the concentrations in the mut/mut group. Furthermore, the patients who responded biochemically to riboflavin were among the patients with the lowest blood FAD concentrations. Finally, these patients had a significantly higher increase in blood FAD compared with the biochemically nonresponding patients. Our results suggest that a relatively low FAD status may be involved in the expression of biochemical features of SCADD. This leads us to hypothesize that individuals with SCADD with a mut/mut genotype will be identified by biochemical screening of urine and/or plasma irrespective of their FAD concentrations, 
van MALDEGEM $E T A L$.

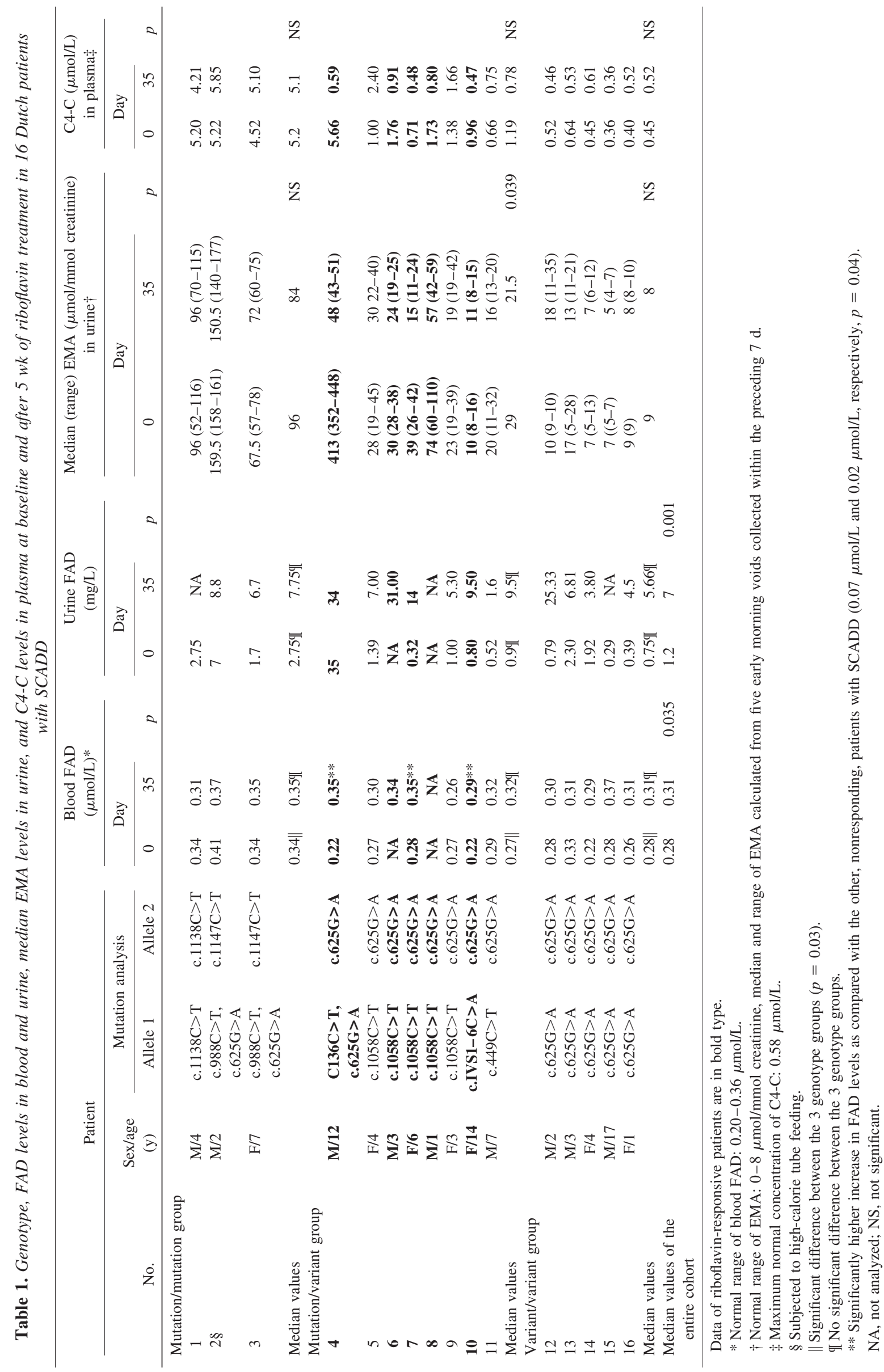


Table 2. Baseline clinical phenotype and changes in the clinical conditions of 16 Dutch SCADD patients after 5 wk of riboflavin treatment

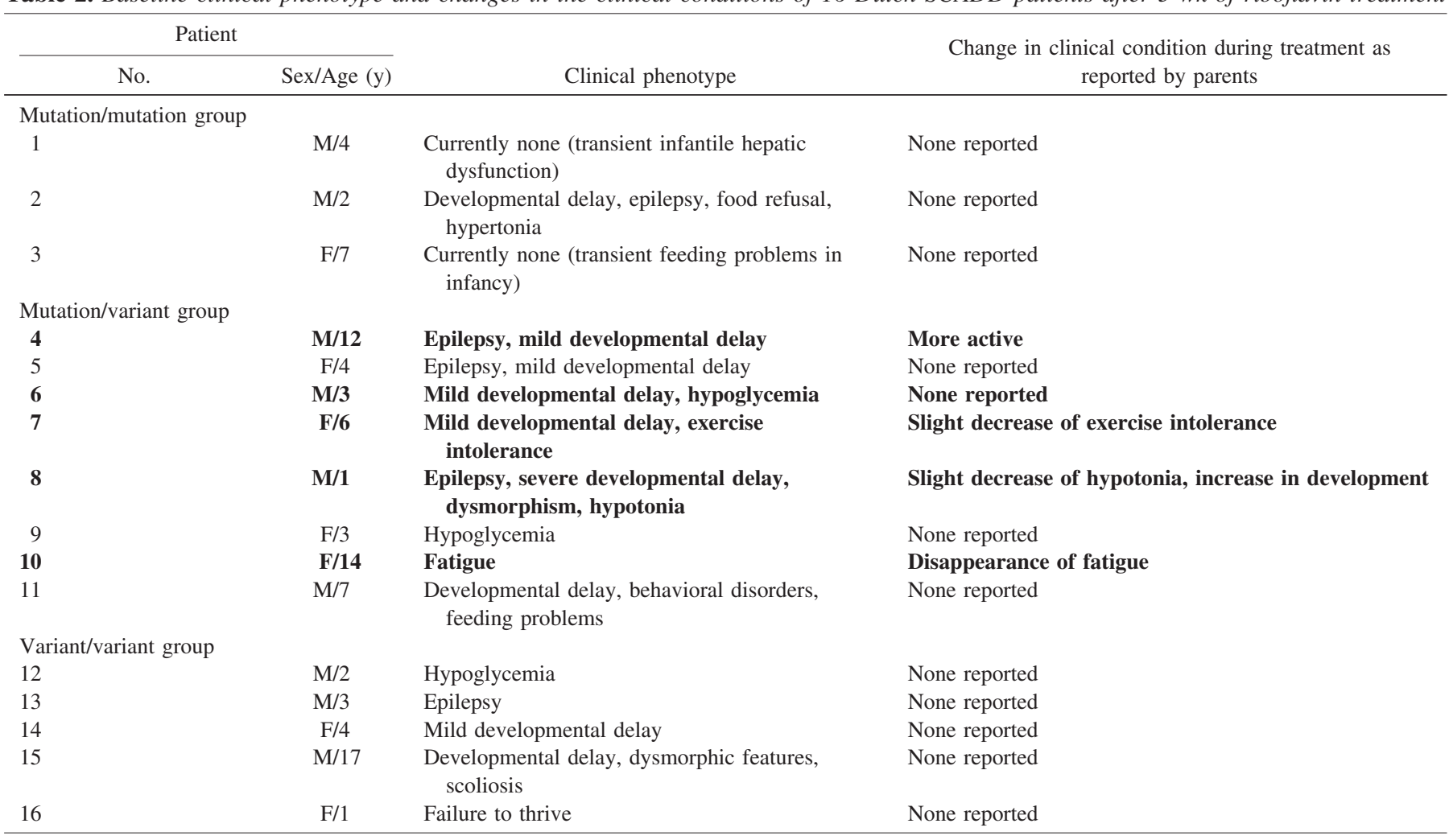

Data of riboflavin-responsive patients (see Table 1) are in bold type.

whereas individuals with SCADD with either a mut/var or a var/var genotype may only be identified by metabolic screening if their FAD levels are low, albeit still in the normal range. In these latter groups, relatively low FAD levels may result in a further decrease of SCAD activity resulting in the characteristic biochemical signs of SCADD. This hypothesis is supported by our observation that patients 4,7 , and 10 (all with a mut/var genotype) achieved nearly normal $\mathrm{C} 4-\mathrm{C}$ concentrations during riboflavin treatment. These findings suggest that patients with these SCADD genotypes may not have been identified by screens for increased $\mathrm{C} 4-\mathrm{C}$ concentrations, the method applied for newborn screening for SCADD, when their FAD levels were high. The number of patients in our study was too small to fully test this hypothesis. It might be of interest to assess the FAD status of individuals with SCADD identified by newborn screening in future studies.

Four of the five patients who responded biochemically to riboflavin showed a slight clinical improvement as reported by their parents. As their initial FAD status was within the normal range and because they had no signs or symptoms specific for riboflavin deficiency, the reported clinical response cannot be explained by the correction of true riboflavin deficiency. The observed clinical improvement may, however, be explained by a placebo effect. This is supported by the observation that none of these patients reported any deterioration in their clinical condition after cessation of riboflavin therapy during a follow-up period of $2 \mathrm{y}$.

None of the patients who were homozygous for the ACADS c. $625 \mathrm{G}>\mathrm{A}$ variant and without an additional $A C A D S$ mutation demonstrated a biochemical improvement while on riboflavin treatment. However, the biochemical abnormalities characteristic for SCADD were only mild at baseline in most of these patients. All patients responding to riboflavin had a mut/var genotype. Our study failed to reveal a correlation between a specific $A C A D S$ mutation and responsiveness to riboflavin treatment (Table 1). This implies that the mut/var genotype may be related to functional SCADD based on decreased FAD affinity or SCAD protein instability associated with the presence of the c.625G $>$ A variant. A low FAD status in combination with the c. $625 \mathrm{G}>\mathrm{A}$ variant may thus be the determinant factors for riboflavin responsiveness.

Although we demonstrated that high-dose riboflavin treatment leads to biochemical improvement in a subgroup of patients with SCADD, it is not clear that it leads to any improvement in clinical disease. This can only be addressed by a properly conducted and preferentially blinded trial of riboflavin in patients with a mut/var genotype, focusing on the clinical efficacy of such an intervention. Such a treatment could be especially effective in those patients who have a relatively low FAD status at baseline. Riboflavin cannot be recommended as a general treatment in SCADD.

Acknowledgments. We thank the patients with SCADD and their parents for their participation in this study; Thessa Westphal and Klaske Honig for their excellent assistance during all the performed tests; and J.H. van der Lee of the Department of Pediatric Clinical Epidemiology for assistance on statistical analyses. 


\section{REFERENCES}

1. Amendt BA, Greene C, Sweetman L, Cloherty J, Shih V, Moon A, Teel L, Rhead WJ 1987 Short-chain acyl-coenzyme A dehydrogenase deficiency. Clinical and biochemical studies in two patients. J Clin Invest 79:1303-1309

2. Waisbren SE, Levy HL, Noble M, Matern D, Gregersen N, Pasley K, Marsden D 2008 Short-chain acyl-CoA dehydrogenase (SCAD) deficiency: an examination of the medical and neurodevelopmental characteristics of 14 cases identified through newborn screening or clinical symptoms. Mol Genet Metab 95:39-45

3. Pedersen CB, Kolvraa S, Kolvraa A, Stenbroen V, Kjeldsen M, Ensenauer R, Tein I, Matern D, Rinaldo P, Vianey-Saban C, Ribes A, Lehnert W, Christensen E, Corydon TJ, Andresen BS, Vang S, Bolund L, Vockley J, Bross P, Gregersen N 2008 The ACADS gene variation spectrum in 114 patients with short-chain acylCoA dehydrogenase (SCAD) deficiency is dominated by missense variations leading to protein misfolding at the cellular level. Hum Genet 124:43-56

4. Baerlocher KE, Steinmann B, Aguzzi A, Krahenbuhl S, Roe CR, Vianey-Saban C 1997 Short-chain acyl-CoA dehydrogenase deficiency in a 16-year-old girl with severe muscle wasting and scoliosis. J Inherit Metab Dis 20:427-431

5. van Maldegem BT, Duran M, Wanders RJ, Niezen-Koning KE, Hogeveen M, Ijlst L, Waterham HR, Wijburg FA 2006 Clinical, biochemical, and genetic heterogeneity in short-chain acyl-coenzyme A dehydrogenase deficiency. JAMA 296:943-952

6. Koeberl DD, Young SP, Gregersen NS, Vockley J, Smith WE, Benjamin DK Jrs, An Y, Weavil SD, Chaing SH, Bali D, McDonald MT, Kishnani PS, Chen YT, Millington DS 2003 Rare disorders of metabolism with elevated butyryl- and isobutyryl-carnitine detected by tandem mass spectrometry newborn screening. Pediatr Res 54:219-223

7. Kurian MA, Hartley L, Zolkipli Z, Little MA, Costigan D, Naughten ER, Olpin S, Muntoni F, King MD 2004 Short-chain acyl-CoA dehydrogenase deficiency associated with early onset severe axonal neuropathy. Neuropediatrics 35:312-316

8. Bhala A, Willi SM, Rinaldo P, Bennett MJ, Schmidt-Sommerfeld E, Hale DE 1995 Clinical and biochemical characterization of short-chain acyl-coenzyme A dehydrogenase deficiency. J Pediatr 126:910-915

9. Birkebaek NH, Simonsen H, Gregersen N 2002 Hypoglycaemia and elevated urine ethylmalonic acid in a child homozygous for the short-chain acyl-CoA dehydrogenase $625 \mathrm{G}>$ A gene variation. Acta Paediatr 91:480-482

10. Corydon MJ, Vockley J, Rinaldo P, Rhead WJ, Kjeldsen M, Winter V, Riggs C, Babovic-Vuksanovic D, Smeitink J, De JJ, Levy H, Sewell AC, Roe C, Matern D, Dasouki M, Gregersen N 2001 Role of common gene variations in the molecular pathogenesis of short-chain acyl-CoA dehydrogenase deficiency. Pediatr Res 49:18-23

11. Gregersen N, Winter VS, Corydon MJ, Corydon TJ, Rinaldo P, Ribes A, Martinez G, Bennett MJ, Vianey-Saban C, Bhala A, Hale DE, Lehnert W, Kmoch S, Roig M, Riudor E, Eiberg H, Andresen BS, Bross P, Bolund LA, Kolvraa S 1998 Identification of four new mutations in the short-chain acyl-CoA dehydrogenase (SCAD) gene in two patients: one of the variant alleles, $511 \mathrm{C}->\mathrm{T}$, is present at an unexpectedly high frequency in the general population, as was the case for $625 \mathrm{G}-$ $>\mathrm{A}$, together conferring susceptibility to ethylmalonic aciduria. Hum Mol Genet $7: 619-627$

12. Dawson DB, Waber L, Hale DE, Bennett MJ 1995 Transient organic aciduria and persistent lacticacidemia in a patient with short-chain acyl-coenzyme A dehydrogenase deficiency. J Pediatr 126:69-71

13. Sewell AC, Herwig J, Bohles H, Rinaldo P, Bhala A, Hale DE 1993 A new case of short-chain acyl-CoA dehydrogenase deficiency with isolated ethylmalonic aciduria. Eur J Pediatr 152:922-924

14. Dietzen DJ, Rinaldo P, Whitley RJ, Rhead WJ, Hannon WH, Garg UC, Lo SF, Bennett MJ 2009 National academy of clinical biochemistry laboratory medicine practice guidelines: follow-up testing for metabolic disease identified by expanded newborn screening using tandem mass spectrometry; executive summary. Clin Chem 55:1615-1626

15. National Newborn Screening and Genetics Resource Center (NNSGRC) 2008 National Newborn Screening Status Report. Available at: http://genes-rus.uthscsa.edu/nbsdisorders.pdf. Accessed October 10, 2009
16. Naito E, Indo Y, Tanaka K 1990 Identification of two variant short chain acylcoenzyme A dehydrogenase alleles, each containing a different point mutation in a patient with short chain acyl-coenzyme A dehydrogenase deficiency. J Clin Invest 85:1575-1582

17. Seidel J, Streck S, Bellstedt K, Vianey-Saban C, Pedersen CB, Vockley J, Korall H, Roskos M, Deufel T, Trefz KF, Sewell AC, Kauf E, Zintl F, Lehnert W, Gregersen N 2003 Recurrent vomiting and ethylmalonic aciduria associated with rare mutations of the short-chain acyl-CoA dehydrogenase gene. J Inherit Metab Dis 26:37-42

18. Bok LA, Vreken P, Wijburg FA, Wanders RJ, Gregersen N, Corydon MJ, Waterham HR, Duran M 2003 Short-chain acyl-CoA dehydrogenase deficiency: studies in a large family adding to the complexity of the disorder. Pediatrics 112:1152-1155

19. Maldegem BT, Waterham HR, Duran M, Vlies M, Woerden CS, Bobu LL, Wanders RJ, Wijburg FA 2005 The $625 \mathrm{G}>\mathrm{A}$ SCAD gene variant is common but not associated with increased C4-carnitine in newborn blood spots. J Inherit Metab Dis 28:557-562

20. Nagan N, Kruckeberg KE, Tauscher AL, Bailey KS, Rinaldo P, Matern D 2003 The frequency of short-chain acyl-CoA dehydrogenase gene variants in the US population and correlation with the C(4)-acylcarnitine concentration in newborn blood spots. Mol Genet Metab 78:239-246

21. Gregersen N, Andresen BS, Bross P 2000 Prevalent mutations in fatty acid oxidation disorders: diagnostic considerations. Eur J Pediatr 159:S213-S218

22. Dainty JR, Bullock NR, Hart DJ, Hewson AT, Turner R, Finglas PM, Powers HJ 2007 Quantification of the bioavailability of riboflavin from foods by use of stable-isotope labels and kinetic modeling. Am J Clin Nutr 85:1557-1564

23. Henriques BJ, Rodrigues JV, Olsen RK, Bross P, Gomes CM 2009 Role of flavinylation in a mild variant of multiple acyl-CoA dehydrogenation deficiency: a molecular rationale for the effects of riboflavin supplementation. J Biol Chem 284:4222-4229

24. Saijo T, Tanaka K 1995 Isoalloxazine ring of FAD is required for the formation of the core in the Hsp60-assisted folding of medium chain acyl-CoA dehydrogenase subunit into the assembly competent conformation in mitochondria. J Biol Chem 270:1899-1907

25. Nagao M, Tanaka K 1992 FAD-dependent regulation of transcription, translation, post-translational processing, and post-processing stability of various mitochondrial acyl-CoA dehydrogenases and of electron transfer flavoprotein and the site of holoenzyme formation. J Biol Chem 267:17925-17932

26. Powers HJ 2003 Riboflavin (vitamin B-2) and health. Am J Clin Nutr 77:1352-1360

27. Bates CJ, Powers HJ 1985 A simple fluorimetric assay for pyridoxamine phosphate oxidase in erythrocyte haemolysates: effects of riboflavin supplementation and of glucose 6-phosphate dehydrogenase deficiency. Hum Nutr Clin Nutr 39:107-115

28. Boisvert WA, Castaneda C, Mendoza I, Langeloh G, Solomons NW, Gershoff SN, Russell RM 1993 Prevalence of riboflavin deficiency among Guatemalan elderly people and its relationship to milk intake. Am J Clin Nutr 58:85-90

29. Hoey L, McNulty H, Strain JJ 2009 Studies of biomarker responses to intervention with riboflavin: a systematic review. Am J Clin Nutr 89:1960S-1980S

30. Kmoch S, Zeman J, Hrebicek M, Ryba L, Kristensen MJ, Gregersen N 1995 Riboflavin-responsive epilepsy in a patient with SER209 variant form of short-chain acyl-CoA dehydrogenase. J Inherit Metab Dis 18:227-229

31. Speek AJ, van Schaik F, Schrijver J, Schreurs WH 1982 Determination of the B2 vitamer flavin-adenine dinucleotide in whole blood by high-performance liquid chromatography with fluorometric detection. J Chromatogr 228:311-316

32. Vreken $P$, van Lint AE, Bootsma AH, Overmars H, Wanders RJ, van Gennip AH 1999 Quantitative plasma acylcarnitine analysis using electrospray tandem mass spectrometry for the diagnosis of organic acidaemias and fatty acid oxidation defects. J Inherit Metab Dis 22:302-306

33. Bikker H, Bakker HD, Abeling NG, Poll-The BT, Kleijer WJ, Rosenblatt DS, Waterham HR, Wanders RJ, Duran M 2006 A homozygous nonsense mutation in the methylmalonyl-CoA epimerase gene (MCEE) results in mild methylmalonic aciduria. Hum Mutat 27:640-643

34. Zempleni J, Galloway JR, McCormick DB 1996 Pharmacokinetics of orally and intravenously administered riboflavin in healthy humans. Am J Clin Nutr 63:54-66 